\title{
Validated LC-MS/MS Method for the Determination of Rosuvastatin in Human Plasma: Application to a Bioequivalence Study in Chinese Volunteers
}

\author{
Dujuan Zhang ${ }^{1,2}$, Jing Zhang ${ }^{2}$, Xiaoyan Liu ${ }^{2}$, Chunmin Wei ${ }^{2}$, Rui Zhang ${ }^{2}$, Haojing Song ${ }^{2}$, Han Yao ${ }^{2}$, \\ Guiyan Yuan", Benjie Wang ${ }^{2}$, Ruichen Guo ${ }^{2 *}$ \\ ${ }^{1}$ School of Pharmacy, Shandong University, Jinan, China, ${ }^{2}$ Institute of Clinical Pharmacology, Qilu Hospital of Shandong University, \\ Jinan, China. \\ Email: *grc7636@126.com
}

Received July $28^{\text {th }}, 2011$; revised August $26^{\text {th }}, 2011$; accepted September $20^{\text {th }}, 2011$.

\begin{abstract}
A sensitive and selective liquid chromatography-electrospray ionization-tandem mass spectrometry (LC-MS/MS) method was developed and validated for the determination of rosuvastatin in human plasma using gliclazide as an internal standard (IS). Rosuvastatin and gliclazide in plasma were extracted with ethyl acetate, separated on a C18 reversed phase column, eluted with mobile phase of acetonitrile-methanoic acid $(0.1 \%)(60: 40, v / v)$, ionized by positive ion pneumatically assisted electrospray and detected in the multi-reaction monitoring mode using precursor $\rightarrow$ product ions of $\mathrm{m} / \mathrm{z} 482.1 \rightarrow 258.1$ for rosuvastatin and $\mathrm{m} / \mathrm{z} 324.2 \rightarrow 127.2$ for IS, respectively. The calibration curve was linear $\left(r^{2}>0.99, n=5\right)$ over the concentration range of $0.1-60 \mathrm{ng} / \mathrm{mL}$. The specificity, matrix effect, recovery, sensitivity, linearity, accuracy, precision, and stabilities were validated for rosuvastatin in human plasma. In conclusion, the validation results showed that this method was sensitive, economical and less toxic and it can successfully fulfill the requirement of bioequivalence study of rosuvastatin calcium tablets in Chinese healthy volunteers.
\end{abstract}

Keywords: Rosuvastatin, Bioequivalence, LC-MS/MS, Pharmacokinetics

\section{Introduction}

Rosuvastatin (Figure 1) is a hydroxyl-methyl-glutaryl coenzyme A (HMG-CoA) reductase inhibitor used in the treatment of patients with dyslipidemia [1]. Rosuvastatin has been reported to reduce serum levels of low-density lipoprotein cholesterol [2], accompanied by increases in high-density lipoprotein cholesterol [3] and reductions in triglycerides [4]. The population pharmacokinetic study revealed that plasma exposure to rosuvastatin was significantly higher in Asian subjects than in White subjects living in the same environment [5].

In recent years, several methods have been reported for the quantification of rosuvastatin in plasma. Kumar TR et al published a HPLC-UV method for the determination of rosuvastatin in rat plasma [6]. Some LC-MS/ MS methods were developed [7-9], but they adopted either ethyl ether as extraction solvent [7,8], which was toxic and difficult to quantitate, or SPE [9], which was relatively expensive, especially for the bioequivalence study where a large number of samples were to be collected and treated. Simultaneous quantitations of rosuvastatin and fenofibric acid [10] or other coadministrated drugs $[11,12]$ in biological samples were also proposed by some literatures, using HPLC with ultraviolet detection $[11,12]$ or LC-MS/MS [10], but the lowest detection limit of these methods was $0.6 \mathrm{ng} / \mathrm{mL}$, not sensitive enough to meet the requirement for rosuvastation determination in plasma. It was therefore necessary to develop a more optimized LC-MS/MS method for the quantitation of rosuvastatin in plasma.

In this paper, a sensitive and selective LC-MS/MS method has been developed and used to evaluate the bioequivalence of two rosuvastatin calcium tablets in healthy Chinese volunteers. 


\section{Experimental}

\subsection{Chemicals and Instrumentation}

Rosuvastatin (lot no. 091201, purity 98.6\%) was obtained from Shandong Dyne Marine Organism Pharmaceutical Co., Ltd. Gliclazide standard (Figure 1, lot no. 100269-9701) was obtained from National Institute for the Control of Pharmaceutical and Biological Products. Methanol, acetonitrile and ethyl acetate were all of HPLC grade, from J. T. BAKER. Methanoic acid and hydrochloric acid were analytical grade, from Sinopharm Chemical Reagent Co., Ltd. Blank human plasma was provided by Shandong blood center (P. R. of China). The test formulation (lot no. 100102) was manufactured by Shandong Dyne Marine Organism Pharmaceutical Co., Ltd. The reference formulation (lot no. GF550) was donated by IPR Pharmaceuticals, Inc.

Determination of rosuvastatin in human plasma was performed with an Agilent 1200 series HPLC and an Agilent 6410 Triple Quadrupole mass spectrometer equipped with an electrospray ionization source (Agilent Technologies, USA).

\subsection{LC-MS/MS Conditions}

The chromatographic separation was achieved on a Diamonsil C18 column $(150 \mathrm{~mm} \times 4.6 \mathrm{~mm}, 5 \mu \mathrm{m})$ at $30^{\circ} \mathrm{C}$ maintained with a thermostated column oven. The mobile phase consisting of acetonitrile-methanoic acid $(0.1 \%)(60: 40, \mathrm{v} / \mathrm{v})$ was employed at a flow rate of 0.8 $\mathrm{mL} / \mathrm{min}$. The injection volume was $20 \mu \mathrm{L}$.

Mass spectrometric analysis was performed in the positive ion MRM mode by monitoring ion transitions at $\mathrm{m} / \mathrm{z} 482.1 \rightarrow 258.1$ for rosuvastatin and $\mathrm{m} / \mathrm{z} 324.2 \rightarrow$ 127.2 for IS (Figure 2), with spray gas pressure of 350 $\mathrm{Pa}$, protective air of nitrogen gas at a flow rate of $9 \mathrm{~L} / \mathrm{min}$, dwell time of $200 \mathrm{~ms}$, capillary voltage of $4000 \mathrm{~V}$, fragment electric voltage of $140 \mathrm{~V}$ for rosuvastatin and 100 $\mathrm{V}$ for IS, and collision energy of $40 \mathrm{eV}$ for rosuvastatin and $20 \mathrm{eV}$ for IS. All data were acquired employing Agilent 6410 Quantitative Analysis version analyst data processing software.

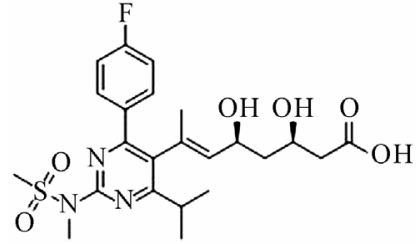

Rosuvastatin

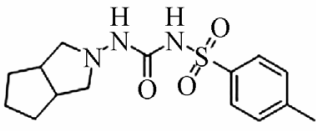

Gliclazide
Figure 1. The chemical structure of rosuvastatin and gliclazide.
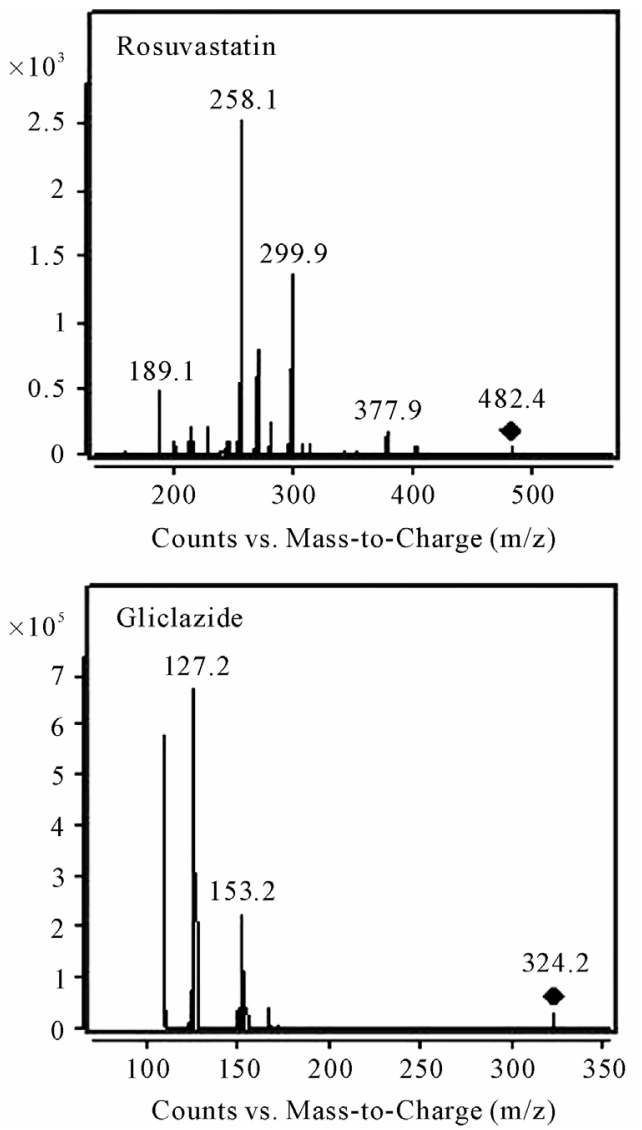

Figure 2. Product ion spectrum of rosuvastatin and gliclazide (IS).

\subsection{Preparation of Standard Solutions, Calibration and Quality Control Samples}

Rosuvastatin standard was accurately weighted and dissolved in methanol achieving concentration of $0.1 \mathrm{mg} / \mathrm{mL}$ stock solution, and further diluted with mobile phase obtaining 1, 10, 100, $1000 \mathrm{ng} / \mathrm{mL}$ working solutions, respectively. The stock solution of IS of $1 \mathrm{mg} / \mathrm{mL}$ was diluted with mobile phase to obtain the working solution of 10 $\mathrm{ng} / \mathrm{mL}$. All were stored in the refrigerator $\left(4^{\circ} \mathrm{C}\right)$, and equilibrated to room temperature before use (approximately 15 minutes).

Rosuvastatin calibration of $0.1,0.5,2,5,10,20$ and 60 $\mathrm{ng} / \mathrm{mL}$ and quality control (QC) of $0.2,5$ and $50 \mathrm{ng} / \mathrm{mL}$ in blank human plasma were prepared by spiking with rosuvastatin and IS working solutions and used for method validation, standard curve or quality control during run of analysis, and all were disposed with the established procedure.

\subsection{Plasma Disposition}

$30 \mu \mathrm{L}$ of the IS $(10 \mathrm{ng} / \mathrm{mL}$ gliclazide in mobile phase) 
was mixed with $0.5 \mathrm{~mL}$ plasma sample, then $100 \mu \mathrm{L}$ hydrochloric acid $(0.1 \mathrm{~mol} / \mathrm{L}$ in water) and $4 \mathrm{~mL}$ ethyl acetate were added, vortex-mixed for $2 \mathrm{~min}$, and centrifuged at $5000 \mathrm{rpm}$ for $5 \mathrm{~min}$. The organic phase was transferred to a clean tube and evaporated to dryness under gentle stream of nitrogen gas at $40^{\circ} \mathrm{C}$. The residue was reconstituted with $100 \mu \mathrm{L}$ mobile phase, and $20 \mu \mathrm{L}$ was injected onto the LC-MS/MS for analysis.

\subsection{Method Validation}

The method was validated for specificity, matrix effect, recovery, sensitivity, linearity, precision, accuracy, and stability according to the US Food and Drug Administration (FDA) guidelines for the validation of bioanalytical methods [13].

The specificity of the method was evaluated by comparing chromatograms of blank plasma, rosuvastain and IS standard, blank plasma spiked with rosuvastain and IS, plasma sample from a volunteer after administration of rosuvastatin calcium tablet.

Blank biological samples were extracted and then spiked with rosuvastatin at three concentration levels $(0.2$ $\mathrm{ng} / \mathrm{mL}$ as low, $5 \mathrm{ng} / \mathrm{mL}$ as medium and $50 \mathrm{ng} / \mathrm{mL}$ as high) and IS in five replicates, respectively, to evaluate the matrix effects of plasma. The corresponding peak areas were then compared to those of standard solutions, and peak area ratio is defined as the matrix effect.

The extraction recoveries were evaluated by comparing peak areas of analytes in spiked biological samples with those of samples to which the analytes had been added after extraction. Five replicates of each QC level were disposed with the established extraction procedure.

The lower limit of quantization (LLOQ) was evaluated by analyzing five replicates of spiked plasma samples at the concentration of $0.1 \mathrm{ng} / \mathrm{mL}$.

The calibration curve was prepared by analyzing spiked calibration samples at 7 different concentration levels on each day of analysis, typically described by equation $\mathrm{y}=$ $\mathrm{ax}+\mathrm{b}$, where $\mathrm{y}$ corresponds to the peak-area ratio and $\mathrm{x}$ to the concentration ratio of rosuvastatin to IS. The linearity of calibration curve was assessed by linear regression with a weighting factor of the reciprocal of the concentration squared $\left(1 / \mathrm{x}^{2}\right)$.

Accuracy and precision were assessed by the determining of QC samples with five replicates for each concentration level on the same day or on three consecutive days. Precision was expressed by coefficient of variation (RSD) and accuracy by relative error (RE).

The stabilities of rosuvastatin in plasma samples at different concentrations were examined under different study conditions; i.e. storing at $-20^{\circ} \mathrm{C}$ for 45 days. Freeze/ thaw stability was determined after freezing $\left(-20^{\circ} \mathrm{C}\right)$ and thawing $\left(25^{\circ} \mathrm{C}\right) \mathrm{QC}$ samples for two cycles. Stability of post-extracted samples in the HPLC auto-sampler at room temperature for 7 hours was also observed.

\subsection{Bioequivalence Study Design}

The study was approved by the Ethics Committee of Qilu Hospital, Shandong University according to Declaration of Helsinki. Twenty healthy young male Chinese volunteers with age of $(24.6 \pm 1.4)$ years, weight of $(64.9 \pm 7.5)$ $\mathrm{kg}$, height of $(173.7 \pm 4.9) \mathrm{cm}$ and body mass index (BMI) of $(21.5 \pm 1.9) \mathrm{kg} / \mathrm{m}^{2}$ were screened to participate and signed Informed Consent Form. $10 \mathrm{mg}$ rosuvastatin tablet was orally administered with $200 \mathrm{~mL}$ of warm water after an overnight fast (10 hours). Standard meals or a given amount of water were provided 4 hours after administration in the study, and no coffee, smoke, chocolate or other food was allowed. All volunteers were under supervision of physicians for any possible adverse events.

Blood samples were collected before and 0.5, 1.0, 2.0, 3.0, 4.0, 5.0, 6.0, 8.0, 10.0, 12.0, 24.0, 36.0, 48.0, 72.0 hours after administration. Blood was centrifuged immediately at $5000 \mathrm{rpm}$ for $5 \mathrm{~min}$, and plasma was transported into two EP tubes equally, clearly labeled and stored at $-20^{\circ} \mathrm{C}$ pending analysis. All plasma samples of 20 volunteers were determined and the pharmacokinetic parameters including the maximum plasma concentration $\left(\mathrm{C}_{\max }\right)$, time to maximum plasma concentration $\left(\mathrm{T}_{\max }\right)$, half-life of drug elimination during the terminal phase $\left(\mathrm{t}_{1 / 2}\right)$, area under the plasma concentration-time curve from 0 to last measurable time $\left(\mathrm{AUC}_{0-\mathrm{t}}\right)$ or from 0 to infinity $\left(\mathrm{AUC}_{0-\infty}\right)$ were estimated using non-compartmental model method. $\mathrm{C}_{\max }$ and $\mathrm{T}_{\max }$ were obtained directly from the measured data. $\mathrm{T}_{1 / 2}, \mathrm{AUC}_{0-\mathrm{t}}$ and $\mathrm{AUC}_{0-\infty}$ were calculated using Drug and Statistical SoftwareVersion 2.0 (DAS 2.0, P. R. of China).

\section{Results}

\subsection{Method Validation}

\subsubsection{Specificity}

Typical MRM chromatograms of blank plasma, rosuvastatin and IS standard, blank plasma spiked with rosuvastatin and IS, plasma from a volunteer after administration of rosuvastatin calcium tablet spiked with IS were shown in Figure 3. Retention times of rosuvastatin and IS were 3.0 and $5.1 \mathrm{~min}$, respectively, and overall run time was within $6 \mathrm{~min}$. No significant interfering peak was observed around the rosuvastatin and IS during analysis.

\subsubsection{Matrix Effect and Extraction Recovery}

The mean matrix effect values of rosuvastatin at three concentrations of $0.2 \mathrm{ng} / \mathrm{mL}, 5 \mathrm{ng} / \mathrm{mL}$ and $50 \mathrm{ng} / \mathrm{mL}$ and 


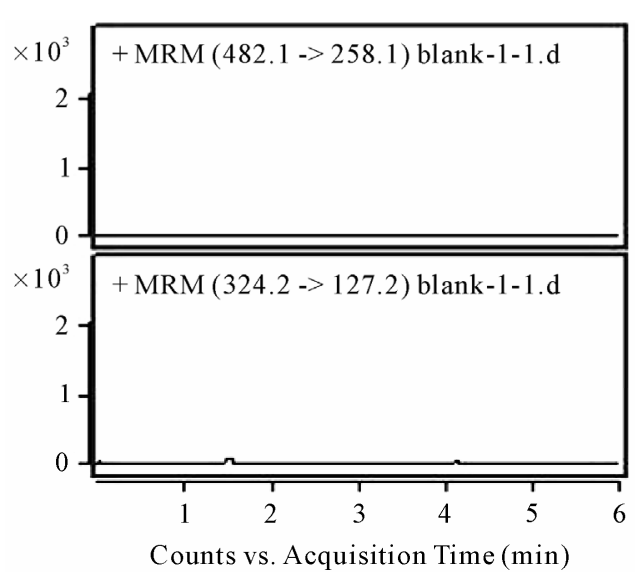

(a)

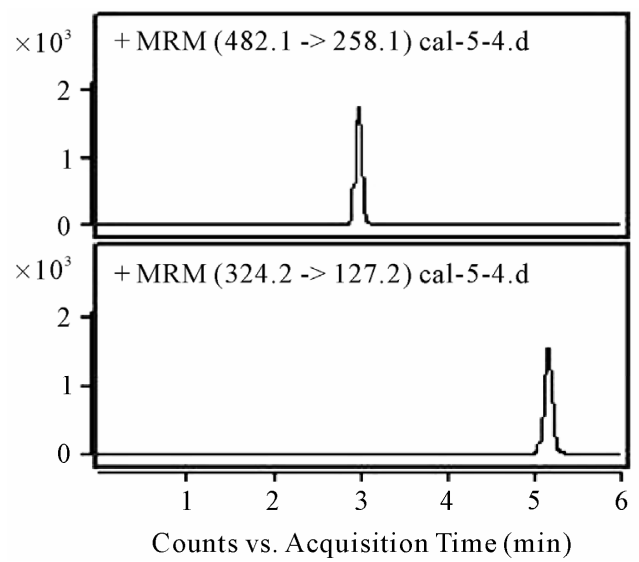

(c)

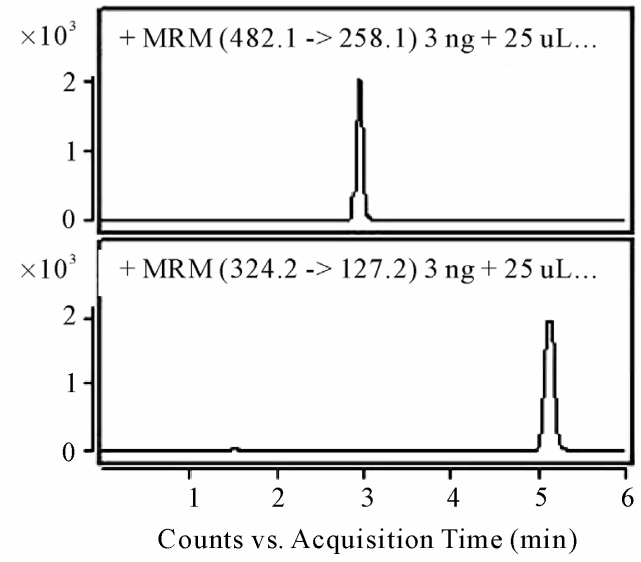

(b)

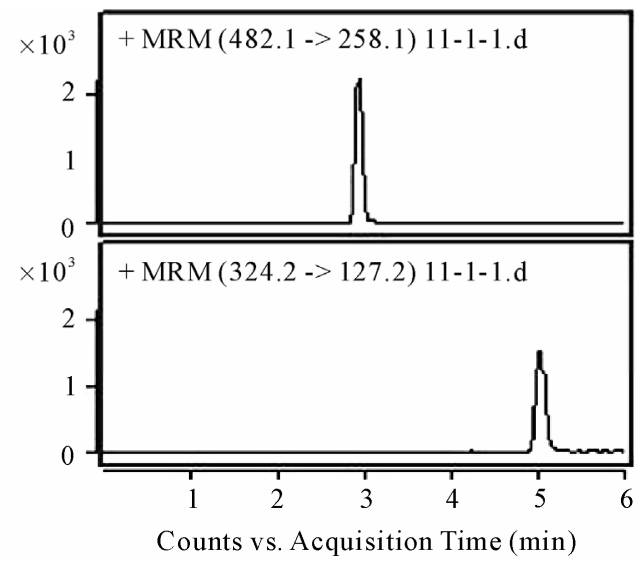

(d)

Figure 3. Typical chromatograms of rosuvastatin and IS in human plasma: blank plasma sample (a); rosuvastatin and IS standard (b); a blank plasma sample spiked with rosuvastatin $(3 \mathrm{ng} / \mathrm{mL})$ and IS (c); a plasma sample of a volunteer at 0.5 hours after administration of $10 \mathrm{mg}$ rosuvastatin calcium tablet (d).

IS at $3 \mathrm{ng} / \mathrm{mL}$ were $(96.41 \pm 6.14) \%,(99.75 \pm 1.43) \%$, $(98.35 \pm 3.01) \%$, and $(88.93 \pm 3.39) \%$, respectively. It demonstrated no matrix significantly affected the determination of rosuvastatin in human plasma.

Mean extraction recoveries of rosuvastatin at three QC levels and IS were $(85.73 \pm 6.62) \%$, $(83.99 \pm 1.52) \%$, $(79.67 \pm 4.13) \%$, and $(75.98 \pm 1.34) \%$, respectively. The results were shown to be consistent, precise and reproducible.

\subsubsection{Calibration Curve and LLOQ}

The calibration curves showed a good linearity in the concentration range of $0.1-60 \mathrm{ng} / \mathrm{mL}$ with correlation coefficient $\left(\mathrm{r}^{2}>0.99\right)$ and the LLOQ for rosuvastatin was proved to be $0.1 \mathrm{ng} / \mathrm{mL}$ with RSD and RE were $4.5 \%$ and $-5.6 \%$, respectively.

\subsubsection{Accuracy and Precision}

The results of intraday and interday precision and accu- racy were shown in Table 1. Both precision values (RSD) were less than $7.7 \%$. Intraday and interday accuracy (RE) ranged from $-10.1 \%$ to $-4.3 \%$ and $-9.5 \%$ to $-2.8 \%$, respectively.

\subsubsection{Stability}

The results of stability of rosuvastatin were shown in Table 2. The data demonstrated that rosuvastain was stable under the indicated conditions for the bioequivalence study. The stock solutions of rosuvastatin and IS were comparable to the freshly prepared ones after storage at $-20^{\circ} \mathrm{C}$ for days and two freeze-thaw cycles.

\subsection{Application to Bioequivalence Study}

The validated method was applied to the bioequivalence study of rosuvastatin in human plasma after a single oral administration of $10 \mathrm{mg}$ rosuvastatin calcium tablet to 20 healthy male volunteers. The mean plasma concentration-time profile and main pharmacokinetic parameters 
Table 1. Interday and intraday precision and accuracy for the determination of rosuvastatin in human plasma $(n=5)$.

\begin{tabular}{ccccccc}
\hline \multirow{2}{*}{$\begin{array}{c}\text { Nominal } \\
\text { Conc. } \\
(\mathrm{ng} / \mathrm{mL})\end{array}$} & \multicolumn{3}{c}{ Mntraday } & \multicolumn{4}{c}{ Interday } \\
\cline { 2 - 7 } MD & $\begin{array}{c}\text { RSD } \\
(\%)\end{array}$ & $\begin{array}{c}\text { RE } \\
(\%)\end{array}$ & Mean $\pm \mathrm{SD}$ & $\begin{array}{c}\text { RSD } \\
(\%)\end{array}$ & $\begin{array}{c}\text { RE } \\
(\%)\end{array}$ \\
\hline 0.2 & $0.19 \pm 0.01$ & 7.7 & -4.3 & $0.19 \pm 0.01$ & 6.3 & -2.8 \\
5 & $4.50 \pm 0.16$ & 3.6 & -10.1 & $4.52 \pm 0.18$ & 4.1 & -9.5 \\
50 & $46.35 \pm 0.99$ & 2.1 & -7.3 & $47.05 \pm 3.34$ & 7.1 & -5.9 \\
\hline
\end{tabular}

Table 2. Stability of rosuvastatin at various conditions $(n=5)$.

\begin{tabular}{ccccc}
\hline Condition & $\begin{array}{c}\text { Nominal Conc. } \\
(\mathrm{ng} / \mathrm{mL})\end{array}$ & Mean $\pm \mathrm{SD}$ & $\mathrm{RSD}(\%)$ & $\mathrm{RE}(\%)$ \\
\hline Fresh samples & 0.2 & $0.21 \pm 0.01$ & 7.0 & 3.4 \\
& 50 & $4.76 \pm 0.29$ & 6.2 & -4.7 \\
& 0.2 & $0.19 \pm 0.01$ & 6.4 & -3.6 \\
$-20^{\circ} \mathrm{C}, 45$ days & 5 & $5.28 \pm 0.27$ & 5.2 & 5.6 \\
& 50 & $45.81 \pm 3.28$ & 7.2 & -8.4 \\
$\begin{array}{c}\text { Two } \\
\text { freeze-thaw } \\
\text { cycles }\end{array}$ & 0.2 & $0.19 \pm 0.02$ & 10.4 & -5.8 \\
& 5 & $5.06 \pm 0.17$ & 3.4 & 1.2 \\
$\begin{array}{c}\text { Post-extracted } \\
\text { samples, } 25^{\circ} \mathrm{C},\end{array}$ & 0.2 & $0.21 \pm 0.01$ & 4.6 & 5.1 \\
$7 \mathrm{~h}$ & 50 & $4.59 \pm 0.19$ & 4.2 & -8.2 \\
\hline
\end{tabular}

were shown in Figure $\mathbf{4}$ and Table 3, respectively.

Bioequivalence of test and reference was evaluated by calculating $90 \%$ confidence intervals $(90 \% \mathrm{CI})$ for the test/reference ratio of logarithmic transformed $\mathrm{C}_{\max }$, $\mathrm{AUC}_{0-\mathrm{t}}$ and $\mathrm{AUC}_{0-\infty}$. The $90 \%$ confidence intervals $(90 \%$ CI) for the test/reference ratio of logarithmic transformed $\mathrm{C}_{\max }, \mathrm{AUC}_{0-\mathrm{t}}$ and $\mathrm{AUC}_{0-\infty}$ were shown in Table 4, and all were within the bioequivalence acceptance range $80 \%$ $125 \%$ adopted by US-FDA [14]. The two rosuvastatin calcium tablets are bioequivalent.

\section{Discussion}

Mass spectrometric detection was carried out on an Agilent 6410 triple quadrupole instrument equipped with an ESI source operated in the positive ion mode. During optimization of the mass spectrometric parameters, strong and stable signals of rosuvastatin and IS can be observed in the form of their $[\mathrm{M}+\mathrm{H}]^{+}$molecular ions with mass to charge ratios of $\mathrm{m} / \mathrm{z} 482.1$ and $\mathrm{m} / \mathrm{z} 324.2$, respectively. Each of the precursor ions was subjected to
Table 3. Main pharmacokinetic parameters of rosuvastatin after a single dose of $10 \mathrm{mg}$ rosuvastatin calcium tablet test and reference $(n=20$, mean $\pm S D)$.

\begin{tabular}{ccc}
\hline Parameter & Test & Reference \\
\hline $\mathrm{t}_{1 / 2} \mathrm{Z}(\mathrm{h})$ & $18.30 \pm 11.34$ & $16.74 \pm 12.70$ \\
$\mathrm{~T}_{\max }(\mathrm{h})$ & $2.55 \pm 0.51$ & $2.50 \pm 0.61$ \\
$\mathrm{AUC}_{0-\mathrm{t}}(\mathrm{ng} \cdot \mathrm{h} / \mathrm{mL})$ & $95.15 \pm 46.68$ & $91.89 \pm 44.34$ \\
$\mathrm{AUC}_{0-\infty}(\mathrm{ng} \cdot \mathrm{h} / \mathrm{mL})$ & $99.10 \pm 48.24$ & $95.24 \pm 45.65$ \\
$\mathrm{C}_{\max }(\mathrm{ng} / \mathrm{mL})$ & $17.52 \pm 8.04$ & $16.53 \pm 7.20$ \\
\hline
\end{tabular}

Table 4. The $90 \%$ Confidence Interval $(90 \% \mathrm{CI})$ for the test/reference ratio of $\mathrm{C}_{\mathrm{max}}, \mathrm{AUC}_{0-\mathrm{t}}$ and $\mathrm{AUC}_{0-\infty}$.

\begin{tabular}{cccc}
\hline Parameter & Mean Ratio (\%) & $90 \% \mathrm{CI}(\%)$ & P-Value \\
\hline $\mathrm{C}_{\max }$ & 105.1 & $99.4-111.1$ & 0.137 \\
$\mathrm{AUC}_{0-\mathrm{t}}$ & 103.2 & $96.2-110.8$ & 0.441 \\
$\mathrm{AUC}_{0-\infty}$ & 103.8 & $96.8-111.4$ & 0.368 \\
\hline
\end{tabular}

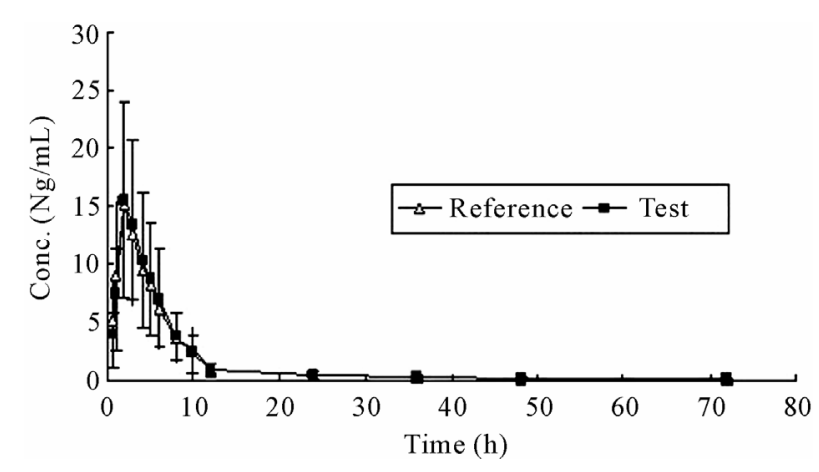

Figure 4. Mean $( \pm \mathrm{SD})$ plasma concentration-time curves of rosuvastatin calcium after a single oral administration of 10 mg rosuvastatin calcium tablet test and reference $(n=20)$.

collision-induced dissociation to determine the resulting product ion. Different collision energy (CE) values (20, $30,40 \mathrm{eV}$ ) for rosuvastatin were tested, and at higher CE $(40 \mathrm{eV})$, a major fragment ion at $\mathrm{m} / \mathrm{z} 258.1$ was formed, giving a considerably better response and a higher signal. Fragmentation of IS produced the most abundant product ion at $\mathrm{m} / \mathrm{z}$ 127.2. Therefore, the ion transitions $\mathrm{m} / \mathrm{z} 482.1$ $\rightarrow 258.1$ and $324.2 \rightarrow 127.2$ were selected for MRM of the rosuvastatin and the IS, respectively.

An ideal IS used to guarantee high accuracy of LCMS/MS assay should track the analyte during the extraction with almost the same recovery of the analyte. It was also eluted close to the analyte on the column and compensated for potential inconsistent response for matrix effects. Several compounds were investigated such as pravastatin sodium, fluvastatin sodium, lorazepam, losar- 
tan potassium, and gliclazide. Significant interfering peaks were observed for pravastatin sodium, fluvastatin sodium and lorazepam during analysis. Different responds were detected for losartan potassium when spiked with purchased blank plasma and plasma samples from volunteers. Gliclazide, with suitable retention time, acceptable matrix effects, and extraction recoveries was selected as internal standard.

Protein precipitation (PPT), liquid-liquid extraction (LLE) and solid-phase extraction (SPE) were tried to obtain a simple and excellent plasma preparation procedure. PPT was easy to dilute the sample and failed to sufficiently remove endogenous interference. The SPE column for solid-phase extraction is relatively expensive for a great quantity of samples. LLE method with various extraction solvents, including chloroform, ethyl acetate, $\mathrm{n}$-hexane, dichloromethane, n-hexane-dichloromethaneisopropanol $(20: 10: 1, \mathrm{v} / \mathrm{v} / \mathrm{v})$ was investigated and evaluated for acceptable extraction recoveries and matrix effect, and ethyl acetate with no-concentration-dependent extraction recovery and acceptable matrix effect was adopted.

In conclusion, the developed method was simple, specific, sensitive, and successfully applied to bioequivalence study of rosuvastatin calcium tablets. The two rosuvastatin calcium tablets were bioequivalent.

\section{Acknowledgements}

The authors appreciated Shandong Dyne Marine Organism Pharmaceutical Co., Ltd, providing financial support and test and reference products.

\section{REFERENCES}

[1] C. I. Carswell, G. L. Plosker and B. Jarvis, "Rosuvastatin," Drugs, Vol. 62, No. 14, 2002, pp. 2075-2085. doi:10.2165/00003495-200262140-00008

[2] D. J. Betteridge and J. M. Gibson, "Effects of Rosuvastatin on Lipids, Lipoproteins and Apolipoproteins in the Dyslipidaemia of Diabetes," Diabetic Medicine, Vol. 24, No. 5, 2007, pp. 541-549. doi:10.1111/j.1464-5491.2007.02095.x

[3] F. McTaggart and P. Jones, "Effects of Statins on HighDensity Lipoproteins: A Potential Contribution to Cardiovascular Benefit," Cardiovascular Drugs and Therapy, Vol. 22, No. 4, 2008, pp. 321-338. doi:10.1007/s10557-008-6113-Z

[4] J. R. Crouse III, "An Evaluation of Rosuvastatin: Pharmacokinetics, Clinical Efficacy and Tolerability," Expert Opinion on Drug Metabolism \& Toxicology, Vol. 4, No. 3, 2008, pp. 287-304. doi:10.1517/17425255.4.3.287

[5] E. Lee, S. Ryan, B. Birmingham, J. Zalikowski, R. March, H. Ambrose, R. Moore, C. Lee, Y. Chen and D. Schneck, "Rosuvastatin Pharmacokinetics and Pharmacogenetics in White and Asian Subjects Residing in the Same Envi- ronment," Clinical Pharmacology \& Therapeutics, Vol. 78 , No. 4, 2005, pp. 330-341. doi:10.1016/j.clpt.2005.06.013

[6] T. R. Kumar, N. R. Shitut, P. K. Kumar, M. C. Vinu, V. V. Kumar, R. Mullangi and N. R. Srinivas, "Determination of Rosuvastatin in Rat Plasma by HPLC: Validation and Its Application to Pharmacokinetic Studies," Biomedical Chromatography, Vol. 20, No. 9, 2006, pp. 881-887. doi: $10.1002 / \mathrm{bmc} .611$

[7] J. Gao, D. Zhong, X. Duan and X. Chen, "Liquid Chromatography/Negative Ion Electrospray Tandem Mass Spectrometry Method for the Quantification of Rosuvastatin in Human Plasma: Application to a Pharmacokinetic Study," Journal of Chromatography B, Vol. 856, No. 1-2, 2007, pp. 35-40. doi:10.1016/j.jchromb.2007.05.012

[8] D. H. Xu, Z. R. Ruan, Q. Zhou, H. Yuan and B. Jiang, "Quantitative Determination of Rosuvastatin in Human Plasma by Liquid Chromatography with Electrospray Ionization Tandem Mass Spectrometry," Rapid Communications in Mass Spectrometry, Vol. 20, No. 16, 2006, pp. 2369-2375. doi: $10.1002 / \mathrm{rcm} .2542$

[9] C. K. Hull, A. D. Penman, C. K. Smith and P. D. Martin, "Quantification of Rosuvastatin in Human Plasma by Automated Solid-Phase Extraction Using Tandem Mass Spectrometric Detection," Journal of Chromatography B, Vol. 772, No. 2, 2002, pp. 219-228. doi:10.1016/S1570-0232(02)00088-0

[10] R. K. Trivedi, R. R. Kallem, R. Mullangi and N. R. Srinivas, "Simultaneous Determination of Rosuvastatin and Fenofibric Acid in Human Plasma by LC-MS/MS with Electrospray Ionization: Assay Development, Validation and Application to a Clinical Study," Journal of Pharmaceutical and Biomedical Analysis, Vol. 39, No. 3-4, 2005, pp. 661-669. doi:10.1016/j.jpba.2005.05.005

[11] S. Vittal, N. R. Shitut, T. R. Kumar, M. C. Vinu, R. Mullangi and N. R. Srinivas, "Simultaneous Quantitation of Rosuvastatin and Gemfibrozil in Human Plasma by HighPerformance Liquid Chromatography and Its Application to a Pharmacokinetic Study," Biomedical Chromatography, Vol. 20, No. 11, 2006, pp. 1252-1259. doi: $10.1002 /$ bmc. 692

[12] Y. Shah, Z. Iqbal, L. Ahmad, A. Khan, M. I. Khan, S. Nazir and F. Nasir, "Simultaneous Determination of rosuvastatin and Atorvastatin in Human Serum Using RPHPLC/UV Detection: Method Development, Validation and Optimization of Various Experimental Parameters," Journal of Chromatography B, Vol. 879, No. 9-10, 2011, pp. 557-563. doi:10.1016/j.jchromb.2011.01.004

[13] US Food and Drug Administration, Center for Drug Evaluation and Research, Guidance for Industry, Bioanalytical Method Validation. http://www.fda.gov/cder/guidance/index.htm

[14] US Food and Drug Administration, "Bioavailability and Bioequivalence Requirements, Abbreviated Applications, Proposed Revisions," Federal Register, Vol. 63, No. 223, 1998, pp. 64222-64228. 